\title{
Toxoplasma gondii Toxolysin 4 contributes to efficient parasite egress from
}

\section{host cells}

3

My-Hang Huynh ${ }^{1}$, Marijo S. Roiko ${ }^{1, a}$, Angelica O. Gomes ${ }^{1, b}$, Ellyn N. Schinke ${ }^{1}$, Aric J. Schultz ${ }^{1, \mathrm{c}}$, Swati Agrawal ${ }^{1, \mathrm{~d}}$, Christine A. Oellig ${ }^{3}$, Travis R. Sexton ${ }^{4}$, Jessica M. Beauchamp ${ }^{1 \mathrm{e}}$, Julie Laliberté ${ }^{1}$, Komagal Kannan Sivaraman ${ }^{3 f}$, Louis B. Hersh², Sheena McGowan ${ }^{3}$, Vern B. Carruthers $^{{ }^{*}}$

${ }^{1}$ Department of Microbiology and Immunology, University of Michigan Medical School, Ann Arbor, MI 48109, USA.

${ }^{2}$ Department of Molecular and Cellular Biochemistry and Center for Structural Biology, University of Kentucky, Lexington, KY 40536, USA

${ }^{3}$ Infection and Immunity Program, Biomedicine Discovery Institute, Department of Microbiology, Monash University, Clayton, Victoria, Australia, 3800.

${ }^{4}$ Department of Cardiology, University of Kentucky, Lexington, KY 40506, USA

*correspondence should be addressed to: V.B.C. (vcarruth@umich.edu)

Running title: $T$. gondii TLN4 facilitates egress

Current Affiliations:

aDepartment of Pathology and Laboratory Services, Altru Health System, 1200 South Columbia Road, Grand Forks, ND 58201

'Departamento de Biologia Estrutural, Universidade Federal do Triângulo Mineiro, Uberaba, MG, 38025440, Brasil

'Lumicks Inc, 800 South Street, Suite 100, Waltham, MA 02453

dDepartment of Biology, University of Mary Washington, Fredericksburg, VA 22401

eDepartment of Microbiology and Immunology, University of North Carolina, Chapel Hill, 125 Mason Farm Road, Chapel Hill, NC 27599 


\section{ABSTRACT}

32 Egress from host cells is an essential step in the lytic cycle of $T$. gondii and other apicomplexan

33 parasites; however, only a few parasite secretory proteins are known to affect this process. The

34 putative metalloproteinase Toxolysin 4 (TLN4) was previously shown to be an extensively

35 processed microneme protein, but further characterization was impeded by the inability to

36 genetically ablate TLN4. Herein we show that TLN4 has the structural properties of an M16

37 family metalloproteinase, that it possesses proteolytic activity on a model substrate, and that

38 genetic disruption of TLN4 reduces the efficiency of egress from host cells. Complementation

39 of the knockout strain with the TLN4 coding sequence significantly restored egress competency,

40 affirming that the phenotype of the $\Delta t \ln 4$ parasite was due to the absence of TLN4. This work

41 identifies TLN4 as the first metalloproteinase and the second microneme protein to function in $T$.

42 gondii egress. The study also lays a foundation for future mechanistic studies defining the

43 precise role of TLN4 in parasite exit from host cells.

\section{IMPORTANCE}

46 After replicating within infected host cells, the single celled parasite Toxoplasma gondii must

47 rupture out of such cells in a process termed egress. Although it is known that T. gondii egress is

48 an active event that involves disruption of host-derived membranes surrounding the parasite,

49 very few proteins that are released by the parasite are known to facilitate egress. In this study we

50 identify a parasite secretory protease that is necessary for efficient and timely egress, thus laying

51 the foundation for understanding precisely how this protease facilitates $T$. gondii exit from host 52 cells. 


\section{INTRODUCTION}

56 Egress by apicomplexan parasites including Toxoplasma gondii and malaria parasites

57 (Plasmodium spp.) is the critical last step in the lytic cycle. Egress liberates the parasite for

58 infection of new cells and releases host cell cytosolic contents, which can activate an

59 inflammatory immune response (1). Inflammation is a hallmark of toxoplasmosis, with

60 associated ocular, neural, cardiac, or respiratory disease, which is especially severe in

61 immunodeficient or congenitally infected individuals. Severe inflammation during toxoplasmosis

62 is linked to the parasite genotype, with highly virulent strains being associated with worse

63 outcome, especially for ocular and congenital infection (2).

Recent focus on egress is elucidating signaling pathways and associated secondary

65 messengers, thus providing a broader understanding of the intricate interplay between the signals

66 involved in this complex cascade of events (recently reviewed in (3). Such studies have

67 solidified roles for cyclic GMP (cGMP) and calcium to respectively stimulate protein kinase G

$68(\mathrm{PKG})(4,5)$ and calcium-dependent protein kinases $(\mathrm{CDPKs})(4,6-8)$, among other targets.

69 Calcium signaling results in the activation of parasite motility and the discharge of apical

70 secretory granules termed micronemes (9-12). While the importance of microneme proteins such

71 as transmembrane adhesins that connect with the actin-myosin motor system to drive gliding

72 motility and active cell invasion have been established, microneme proteins also contribute to

73 active egress via their role in the formation of pores such as those created by Perforin-Like

74 Protein 1 (PLP1) (13-15). Gene knockout studies suggest that PLP1 pore formation disrupts the

75 parasitophorous vacuole membrane (PVM), which encases parasites during intracellular

76 replication. PLP1 deficient parasites are delayed or fail in egress and show a marked loss of

77 virulence in infected mice, implying a link between efficient egress and virulence. Although

78 several proteins released from parasite dense granules (calcium independent secretory organelles 
79 released during parasite replication) have also been implicated in egress (16-20), PLP1 is the

80 only microneme protein known to directly function in egress to date.

Previous work identified a putative metalloprotease Toxolysin 4 (TLN4, TGME49_206510)

82 in a proteomic screen of Toxoplasma secretory products released by extracellular tachyzoites

83 (21). A subsequent study showed that TLN4 is a microneme protein that undergoes extensive

84 proteolytic processing and potentially contributes to parasite fitness, based on loss of TLN4

85 deficient parasites from a mixed population of parasites transfected with a knockout plasmid

86 (22). However, a recent genome-wide CRISPR/Cas9 knockout screen indicated that TLN4 does

87 not contribute substantially to parasite fitness based on its phenotype score of 0.51 (on a scale of

$88-7$ to 3, with lower values indicating lower fitness) (23). TLN4 is a member of the M16

89 metalloproteinase subfamily of so-called "cryptases", which are represented throughout the tree

90 of life and include four TLN genes encoded in the Toxoplasma genome. M16 metalloproteinases

91 are exemplified by the human Insulin-Degrading Enzyme (IDE) and feature a catalytic chamber

92 (or crypt) that accommodates small polypeptides for degradation. Whereas characterization of

93 TLN2 (TGME49_227948) and TLN3 (TGME49_257010) has not been reported, TLN1

94 (TGME49_269885) resides in the parasite secretory rhoptries where it plays an unknown role

95 apart from contributing modestly to growth in vitro (24).

96 Here we show that TLN4 has the structural features of an active metalloproteinase and that it

97 possesses proteolytic activity against a model polypeptide substrate. We also report that TLN4

98 deficient parasites show normal gliding motility, cell invasion, and replication, but have a

99 delayed induced-egress phenotype. Our findings suggest that TLN4 contributes to Toxoplasma

100 egress, identifying it as only the second microneme protein implicated in this event. 
103 TLN4 has the structural features of an active metalloproteinase. TLN4 is a large (2,435 aa)

104 protein that contains multiple domains including one active (A) and three inactive (IA) M16

105 proteinase domains, along with a long C-terminal extension that includes a repeat domain

106 consisting of eight tandem repeats of a 28 aa sequence (Fig. 1A). Structural modeling of the M16

107 proteinase domains (residues 202-1367) using the human insulin degrading enzyme as a template

108 suggests that each domain forms a similar $\alpha \beta$ roll fold, with the domains arranged around a central

109 chamber (Fig. 1B), as expected for an M16 family metalloproteinase (25). When viewed from a

110 perspective of inside the chamber or "crypt", a putative active site is visible (Fig. 1C) with the

111 characteristic $\mathrm{HXXEHX}_{\mathrm{N}} \mathrm{EX}_{6} \mathrm{E}$ binding motif for catalytic $\mathrm{Zn}^{2+}$ arranged on two $\alpha$-helices (Fig.

112 1D) that are separated by 60 amino acids in TLN4 (Fig. 1E). These features are consistent with

113 TLN4 being an active metalloproteinase of the M16 family.

115 Recombinant TLN4 is capable of processing $\boldsymbol{\beta}$-insulin. M16 family proteinases typically act

116 upon peptides and polypeptides that are sufficiently small to fit in the crypt. To determine if TLN4

117 possesses proteolytic activity, we expressed and purified a recombinant form of TLN4 lacking the

118 C-terminal extension. TLN4 $209-1295$ was expressed using a bacterial expression system and was

119 purified and refolded. The purified protein migrated on SDS PAGE as a prominent $130 \mathrm{kDa}$ band

120 and behaved as a monodispersed protein when analyzed by size exclusion chromatography (Fig.

121 2A). Upon incubating recombinant TLN4 with $\beta$-insulin as a model substrate, we observed the

122 generation of several cleavage products (Fig. 2B), which were confirmed by mass spectrometry to

123 originate from $\beta$-insulin (Fig. 2C). Mapping of the cleavage sites on the sequence of $\beta$-insulin

124 revealed a preference for TLN4 cleavage in the central region of the polypeptide but no obvious

125 preference for recognition of specific amino acids. Collectively our findings suggest that TLN4 is

126 an active protease that is capable of processing a model substrate. 
$128 T$ TLN4 is amenable to genetic disruption. In a previous study of TLN4, it was proposed that TLN4

129 contributes to parasite fitness because TLN4-knockout parasites could be detected in a population

130 of transfected RH parasites but were lost through further culturing (22). To enhance the recovery

131 of knockout parasites we utilized RH $\Delta k u 80 \Delta h x g$ parasites (WT hereafter) and transfected them

132 with a hypoxanthine xanthine guanine phosphoribosyl transferase (HXGPRT) selectable cassette

133 flanked by 5' and 3' TLN4 homology regions (Fig. 3A). Individual knock-out parasite clones were

134 tested by PCR for integration of the selectable marker at the 5' end (Fig. 3B, left gel), at the 3' end

135 (Fig 3B, middle gel), and for the absence of the TLN4 gene ( $\Delta t \ln 4)$ (Fig 3B, right gel). We then

136 genetically complemented $\Delta t \ln 4$ parasites by expression of the $T N L 4$ cDNA containing two copies

137 of an HA epitope tag inserted at aa position 847 after the first inactive domain (TLN4-IA ${ }^{1}-\mathrm{HA}_{2}$ )

138 to generate $\triangle t \ln 4 T L N 4$ parasites, which were confirmed by PCR (Fig. 3C). The difference in

139 fragment size in the WT and the $\triangle \operatorname{tn} 4 T L N 4$ is due to the presence of introns in the WT but not in

140 the cDNA of the complement strain. Expression of TLN4 in $\triangle t \ln 4 T L N 4$ parasites was confirmed

141 by fluorescence microscopy (Fig. 3D) and by western blot, with the expected shift in migration of

142 the $\sim 33 \mathrm{kDa}$ processed species due to insertion of HA (Fig. 3E). The loss of the TLN4 protein in

$143 \Delta t \ln 4$ was also confirmed by western blot (Fig. 3E).

145 TLN4 does not contribute to invasion, replication, gliding motility, or virulence. To assess

146 whether TLN4 plays a role in the lytic cycle, plaque assays were performed. Compared to parental

147 parasites, $\Delta t \ln 4$ parasites showed significantly smaller plaques (Fig. 4A,B), suggesting a defect in

148 one or more steps in the parasite lytic cycle. This phenotype that was fully restored in the

149 complement strain. We next tested each step in the lytic cycle to identify the basis of smaller

150 plaques. Parasites deficient in TLN4 showed normal invasion (Fig. 4C), replication at $17 \mathrm{~h}$ and 26 
151 h post-infection (Fig. 4D) and all types of gliding motility were observed (Fig. 4E). Finally, no

152 significant differences were observed in mouse survival of acute infection upon intraperitoneal

153 infection with 10 or 100 tachyzoites (Fig. 4F).

$\Delta t \ln 4$ parasites are defective in efficient egress. The last step of the lytic cycle is egress of the

156 intracellular parasites from host cells, which can be induced by addition of the calcium

157 ionophore A23187. Whereas most wild-type parasites egressed from host cells within 2 min of

158 ionophore addition, significantly fewer $\Delta t \ln 4$ parasites egressed in the allotted time (Fig. 5A).

159 This phenotype is mostly reversed in $\Delta t \ln 4 T L N 4$ parasites. The deficiency in egress was more

160 pronounced when zaprinast, a phosphodiesterase inhibitor, was used as an inducer (Fig. 5B),

161 which may be due to its mode of action. Whereas A23187 overtly elevates cytosolic calcium by

162 mobilizing it from intracellular stores and the medium, zaprinast treatment triggers the release of

163 calcium from intracellular stores via activation of protein kinase $\mathrm{G}$ and inositol triphosphate

164 signaling $(4,26)$. A time-course of egress further illustrated the delay in $\Delta t \operatorname{tn} 4$ parasites, with a

165 statistically significant difference in the time to 50\% egress compared to WT parasites (Fig. 5C).

$166 \Delta p l p 1$ parasites, which were included as a reference strain, showed an expected severe impaired

167 in egress. An alternative and complementary measure of parasite egress is the release of host

168 lactate dehydrogenase (LDH) into the surrounding media due to loss of host cell integrity. After

169 normalizing LDH release to WT parasites, $\Delta t \ln 4$ parasites showed $\sim 45 \%$ release of LDH, while

170 the complement strain nearly restored LDH release to wild-type levels (Fig. 5D). In examining

171 the excreted-secreted-antigen fraction of extracellular parasites, conducted in buffers with neutral

172 or acidic $\mathrm{pH}$ (to mimic PV acidification; (13-15)), there was no change in the processing of

$173 \quad$ PLP1 in the $\Delta t \ln 4$ parasites compared to WT parasites (Fig. 5E). Together these findings suggest

174 a role for TLN4 in T. gondii egress from host cells that is independent of PLP1 processing. 


\section{DISCUSSION}

177 TLN4 was previously shown to be an extensively processed microneme protein (22), but its

178 contribution to the lytic cycle was not determined due to the inability to generate a knockout of

179 this gene in the wildtype RH strain background. In this study we were able to isolate a TLN4

180 knockout strain by utilizing the more genetically tractable RH $\Delta k u 80$ strain. Plaque assays showed

181 that $\Delta t \ln 4$ parasites affected the lytic cycle, based on the smaller plaque sizes observed. Assessing

182 the known steps of the lytic cycle showed that there were no defects in invasion, replication, or

183 types of gliding motility performed by the parasites, nor was there any effect on parasite virulence.

184 The only deficiency observed was in induced egress, assessed by a static egress assay, a time-

185 course of egress, and LDH-release. Complementation of $\Delta t \ln 4$ with a TLN4 construct under the

186 control of the endogenous TLN4 promoter fully restored the plaque defect and largely restored the

187 egress deficiency.

188 In vitro investigation into TLN4 suggests that the protein is an active protease based on

189 cleavage of $\beta$-insulin as a model substrate, supporting a potential proteolytic function within the

190 parasite. Homology modeling indicates that TLN4 likely adopts the typical M16 structure and

191 contains a catalytic chamber or "crypt", which is one of the defining features of the M16 family of

192 metalloproteinases. Studies with other M16 enzymes indicate that the crypt encapsulates and

193 cleaves polypeptides, with the substrate being determined by the size and charge of the crypt as

194 well as the flexibility of the substrate(s) $(27,28)$. Although it is possible that TLN4 plays a direct

195 role in egress by e.g., facilitating the disruption of the PVM, an indirect contribution of TLN4 to

196 egress is also plausible. An indirect role could involve proteolytically activating another parasite

197 protein that contributes to egress or degrading a protein that would otherwise compromise egress.

198 Identifying substrates of TLN4 in future studies might reveal the basis for its contribution to 
parasite exit from host cells.

PLP1 is the only microneme protein identified to date to have a direct role in egress.

201 Interestingly, mice infected with $\Delta t \ln 4$ have no virulence defect, whereas $\Delta p l p 1$ parasites are

202 markedly virulence attenuated. This notable distinction could be because the egress defect of $\Delta t \ln 4$

203 parasites is less pronounced than that for $\Delta p l p 1$ or it could be due to additional roles for PLP1

204 during infection of mice. Future studies identifying other secretory proteins that contribute to 205 egress to varying degrees along with other work defining how PLP1 shapes the outcome of

206 infection should help distinguish between these possibilities.

207 Among the Apicomplexa, the M16A subfamily is constrained to coccidian parasites, including

208 close (e.g., Hammondia hammondia, Neospora caninum) and more distant (Sarcocystis neurona,

209 Eimeria spp., Cryptosporidium spp.) relatives of T. gondii (orthomcl.org). Whereas the genome of

210 T. gondii encodes 11 M16 metalloproteinases, of which 4 belong to the M16A subfamily (29), the

211 genome of the intestinal parasite Cryptosporidium parvum encodes an expanded family of 22 M16

212 metalloproteinases, 18 of which are M16A members. Among these, INS1 was recently shown to

213 be necessary for formation of macrogamonts, the female sexual stage of C. parvum (30). The

214 results of reciprocal BLAST searches indicate that TLN4 is most closely related to C. parvum INS-

$21515\left(\operatorname{cgd} 3 \_4260\right)$ and INS-16 (cgd3_4270), which are closely related orthologs (83\% identical) (30,

216 31). INS-15 localizes to the mid-apical region of $C$. parvum sporozoites and possibly merozoites

217 (31). Antibodies to INS-15 impaired sporozoite cell invasion, suggesting a role in parasite entry

218 into host cells. Future studies involving the targeted deletion of M16A family members in $C$.

219 parvum and other coccidian parasites will be necessary to appreciate further their contributions to 220 parasite infection biology. 
Structural modeling of TLN4. The full amino acid sequence of ME49 strain TLN4 was used to query Phyre2, which identified human insulin degrading enzyme (PDB 2JBU) as the top scoring template. This analysis resulted in a structural model of TLN4 encompassing amino acids 2021367. The structural model was visualized using PyMOL v2.3.2.

Expression and purification of recombinant TLN4. A region encoding domains A to IA-3 of the TLN4 cDNA from RH strain (amino acids 209 - 1295) was amplified with TLN4.625.NdeI.F (5'-GGAATTCCATATGAGAGACACGAGCGCGTACTCGG-3') and TLN4.3885.Not.R (5'AAGGAAAAAAGCGGCCGCGCTAAGCCACCGGAGGCGCTCGAGGAAAGC-3’) primers,

232 and cloned into the bacterial expression vector pET21a, introducing an in-frame C-terminal

233 hexa-Histidine tag to the construct. The E. coli expression construct containing the TLN4 209-1295

234 ORF was verified by DNA sequencing.

The TLN4 209-1295 expression plasmid was transformed into Escherichia coli BL21 Gold cells 236 selected on 2x yeast-tryptone (2YT) broth-agar plates containing $100 \mu \mathrm{g} / \mathrm{mL}$ ampicillin. $20 \mathrm{~mL}$ 237 of 2 YT supplemented with ampicillin was grown to confluence overnight and then added to $1 \mathrm{~L}$ 238 of $2 \mathrm{YT}$ containing ampicillin and incubated with shaking at $37^{\circ} \mathrm{C}$ until an $\mathrm{OD}_{600}$ of $\sim 0.85$ was

239 reached. Cultures were then induced with a final concentration of $1 \mathrm{mM} \mathrm{IPTG}$ overnight at $16^{\circ} \mathrm{C}$.

240 Harvest and cell lysis of the overnight expression culture showed that TLN4 $209-1295$ expressed

241 to high yields but was insoluble. Inclusion bodies were prepared by harvest of the insoluble

242 fraction of the whole cell lysate and resuspension with $30 \mathrm{~mL}$ of wash buffer I (25 mM Tris $\mathrm{pH}$

$2438.0,300 \mathrm{mM} \mathrm{NaCl}, 1 \%$ Triton X-100). The suspension was homogenized until an even

244 consistency was obtained. The inclusions bodies were then washed twice by centrifugation and

245 homogenization in wash buffer I. Three subsequent washes with wash buffer II (25 mM Tris pH

$2468.0,300 \mathrm{mM} \mathrm{NaCl}$ ) were performed to remove the excess Triton X-100. Purified inclusion 
247 bodies were stored at $-80^{\circ} \mathrm{C}$ until required. Proteins were resolubilized in $25 \mathrm{mM}$ Tris $\mathrm{pH} 8.0,250$

$248 \mathrm{mM} \mathrm{NaCl}, 6 \mathrm{M}$ guanidine hydrochloride, $2 \mathrm{mM}$ beta-mercaptoethanol by rotation at $4^{\circ} \mathrm{C}$ before

249 centrifugation. Harvest supernatant was applied to Ni-NTA resin and purified via metal affinity

250 chromatography. Purity of the denatured purified protein was assessed via SDS-PAGE and

251 western blot.

252 Denatured TLN4209-1295 was diluted to a final concentration $<1 \mathrm{mg} / \mathrm{mL}$ and applied

253 dropwise to $100 \mathrm{~mL}$ of refold buffer (50 mM Tris $\mathrm{pH} 8.2,250 \mathrm{mM} \mathrm{NaCl}, 0.5 \mathrm{M}$ arginine, $0.44 \mathrm{M}$

254 sucrose, $4 \mathrm{mM}$ reduced glutathione, $0.4 \mathrm{mM}$ oxidised glutathione, $0.05 \mathrm{mM}$ Tween-20) that was

255 constantly stirring at $4^{\circ} \mathrm{C}$. Refolding proceeded for $4 \mathrm{~h}$ at $4^{\circ} \mathrm{C}$ with gentle stirring. The refolded

256 protein was then dialyzed overnight against $25 \mathrm{mM}$ Tris $\mathrm{pH} 8.0,300 \mathrm{mM} \mathrm{NaCl}$ at $4^{\circ} \mathrm{C}$. Post-

257 dialysis, the protein was concentrated to approximately $0.5 \mathrm{~mL}$ before application to a Superdex

258200 10/300 column for further purification via size-exclusion chromatography.

TLN4 proteolytic activity. Hydrolysis of Insulin B Chain was measured via reverse phase high

261 performance liquid chromatography (HPLC) following incubation of TLN4 $(160 \mu \mathrm{g} / \mathrm{mL})$ with

262 insulin B chain (42 $\mu \mathrm{M}$, Sigma Aldrich) in $10 \mathrm{mM}$ Tris for $88 \mathrm{~h}$. HPLC was carried out in a

263 Vydac C4 HPLC column using a linear gradient from $0.1 \%$ trifluorocacetic acid (TFA) in 95\%

264 water, $5 \%$ acetronitrile to $0.1 \%$ TFA in $50 \%$ acetonitrile $/$ water at a flow rate of $1 \mathrm{~mL} / \mathrm{min}$.

265 Hydrolysis products were detected at $214 \mathrm{~nm}$ and collected for analysis. The obtained peptide

266 products were analyzed on an Applied Biosystems 4800 MALDI TOF/TOF Proteomics Analyzer

267 at the University of Kentucky Proteomics core.

269 Parasite culture, transfection, and selection. T. gondii tachyzoites were maintained by growth

270 on monolayers of human foreskin fibroblasts (HFF) in Dulbecco's Modified Eagles Medium 
271 (DMEM) containing 10\% Cosmic Calf Serum (GIBCO), 2 mM Glutamine, 10 mM HEPES, 50

$272 \mu \mathrm{g} / \mathrm{mL}$ Penicillin/Streptomycin (D10 Complete). To generate the TLN4 KO, a knockout

273 construct (described in (Laliberte \& Carruthers, 2011)) containing $1.5 \mathrm{~kb}$ of TLN4 5' and 3'

274 flanking regions was used and the HXGPRT selectable marker was replaced with the DHFR-TS

275 selectable marker cassette. This construct was transfected into RH $\Delta k u 80 \Delta h x g$ parasites with a

276 BioRad Gene Pulsar II with $1.5 \mathrm{kV}$ voltage, $25 \mu \mathrm{F}$ capacitance and no resistance setting.

277 Pyrimethamine selection was applied the day after transfection and clones were isolated by

278 limited dilution in 96-well plates. Knockout clones were tested for proper 5' integration (a) 5'-

279 agttgcagccagaggcagaagcaagtcc-3' and (b)

280 5'-cagtcagataacaggtgtagcg-3' and for proper 3' integration (c) 5' -gcgggtgacgcagatgtgcgtgtatcc-

281 3'; (d) 5_-gaaaagtgtctgcgtgttagcagc-3. Knockout clones that were complemented with the

282 TLN4.HA construct were identified with primers TLN4.322.F (e)

283 5'GGCTTTTCTGCTTCGTCAAC-3' and TLN4.1910.R (f)

284 (5'AAGAGCAGTGGGCTGAAAAA-3').

285

286 Invasion assays. Invasion assays were performed as previously described (32). Briefly, $1 \times 10^{7}$

287 parasites were used to infect sub-confluent HFF monolayers in 8-well chamber slides for 20 min 288 before fixation with paraformaldehyde. Slides were differentially stained with anti-SAG1 289 antibodies to differentiate attached versus invaded parasites.

291 Egress assays. Induced egress was performed as described previously (15). Briefly, parasites

292 grown in HFFs in an 8-well chamber slide for $30 \mathrm{~h}$ were treated with $1 \%$ dimethyl sulfoxide, 2

$293 \mu \mathrm{M}$ A23187, or $200 \mu \mathrm{M}$ zaprinast in assay buffer (Hanks' buffered salt solution containing $1 \mathrm{mM}$

$294 \mathrm{CaCl}_{2}, 1 \mathrm{mM} \mathrm{MgCl}$, and $10 \mathrm{mM} \mathrm{HEPES}$ ) and incubated for $2 \mathrm{~min}$ in a $37^{\circ} \mathrm{C}$ water bath. Egress 
was stopped by addition of 2x fixative ( $8 \%$ formaldehyde in $1 \mathrm{x}$ PBS). Immunofluorescence was performed with rabbit anti-SAG1 to identify parasites and mouse anti-GRA7 (a generous gift

297 from Peter Bradley) to identify the parasitophorous vacuole membrane. At least 10 fields of view

298 (400x total magnification) per condition were enumerated as occupied or unoccupied.

299 LDH egress assays were performed as previously described (20). Briefly, parasites were

300 grown in HFF monolayers in 96-well plates for $30 \mathrm{~h}$. Wells were washed with Ringer's buffer

301 and then treated with $100 \mu \mathrm{M}$ zaprinast diluted in Ringer's buffer. Plates were incubated at $37^{\circ} \mathrm{C}$

302 for $20 \mathrm{~min}$ before removal and placement on ice. Fifty microliters of the supernatant were

303 removed and centrifuged in a separate round-bottom plate. Thirty microliters of supernatant was

304 subsequently removed and release of lactate dehydrogenase was determined using an LDH

305 Cytotoxicity Colorimetric Assay kit (BioVision).

306 Time-course egress assays were performed as described above, with time points of $0.5,1$,

307 1.5, 2, 2.5, 3 and 4 min following induction with A23187, at which time wells were fixed, and

308 IFA performed with rabbit anti-SAG1 and mouse anti-GRA7.

310 Plaque and replication. Parasites were inoculated into wells of a 6-well plate and allowed to

311 replicate undisturbed for 7 days. The wells were then stained with $0.2 \%$ Crystal violet for 5 min

312 and rinsed with $\mathrm{ddH}_{2} \mathrm{O}$. Images of the wells were scanned, and plaque number and size were

313 analyzed with Image $\mathbf{J}$.

314 For the replication assay, cells of an 8-well chamber slide were inoculated with $1.25 \times 10^{5}$

315 tachyzoites and allowed to invade and grow for $18 \mathrm{~h}$ prior to fixation and indirect

316 immunofluorescence.

318 Live gliding video microscopy. Glass-well dishes (MatTek) were coated with 

were filter-purified and resuspended in HHE (Hank's salt solution, 10mM HEPES, EDTA).

321 Parasites were then added to coated dishes and allowed to settle for $5 \mathrm{~min}$ at room temperature.

322 Dishes were then moved to a $37^{\circ} \mathrm{C}$ chamber with $5 \% \mathrm{CO}_{2}$ and warmed for 5 min prior to starting

323 video recording. Each video consisted of 1 frame/sec recorded for $90 \mathrm{sec}$. Enumeration of the

324 types of gliding motility were carried out by examining the videos in addition to maximum

325 projection images generated by the Zeiss AxioVision software.

327 Mouse infections. All laboratory animal work in this study was carried out in accordance

328 with policies and guidelines specified by the Office of Laboratory Animal Welfare, the US

329 Department of Agriculture, and the American Association for Accreditation of Laboratory

330 Animal Care (AAALAC). The University of Michigan Committee on the Use and Care of

331 Animals (IACUC) approved the animal protocol used for this study (Animal Welfare Assurance

332 A3114-01, protocol PRO00008638). Freshly egressed parasites were filter purified in PBS,

333 washed, counted, and injected intraperitoneally in $200 \mu 1$ of PBS into 6-8-week-old female Swiss

334 Webster mice. The experiment was performed once with 4 groups of 12 mice each infected with

33510 or 100 tachyzoites of WT or $\Delta t \ln 4$. Mice were given water and food ad libitum, monitored

336 twice daily and were humanely euthanized upon showing signs of moribundity.

338 Western blotting. Parasite lysates were generated by filter-purifying parasites followed by

339 centrifugation, $1 \mathrm{x}$ wash with cold PBS, and resuspension in $>90^{\circ} \mathrm{C} 1 \mathrm{x}$ sample buffer. Lysates

340 were boiled for 5 min prior to running on SDS-PAGE gels. Gels were semi-dry electroblotted

341 (Bio-Rad) onto polyvinylidene fluoride membranes and sequentially probed with mouse anti-

342 TLN4 (22) and horse radish peroxidase conjugated goat anti-mouse (Jackson ImmunoResearch). 
343 Bands were revealed by enhanced chemiluminescence with SuperSignal West Pico PLUS

344 Chemiluminescent Substrate (Thermo Fisher Scientific) and documented with a Syngene Pxi

345 imaging system.

347 PLP1 processing. Extracellular parasites ( $\Delta k u 80 \Delta h x g(\mathrm{WT}), \Delta p \operatorname{lp} 1, \Delta t \ln 4$, and $\Delta t \ln 4 T L N 4)$ were

348 resuspended in PBS in either pH 5.4 or 7.4 and ESA was collected. Pellet lysates and ESA were

349 separated on SDS-PAGE gels and membranes were probed with antibodies against rabbit anti-

$350 \quad$ PLP1.

351

352 ACKNOWLEDGEMENTS

353 This work was supported by National Institutes of Health grant R01AI46675 (VBC) and

354 1R01GM130954 and 5P30GM110787 (LBH). AJS received fellowship support from the

355 American Heart Association. We thank Tracey L. Schultz for technical assistance. 
A

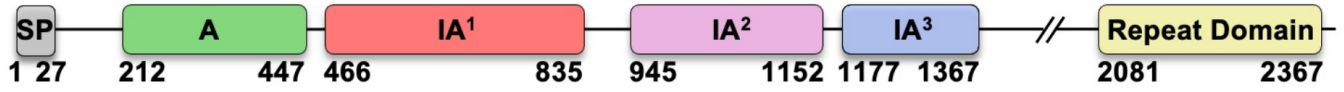

B
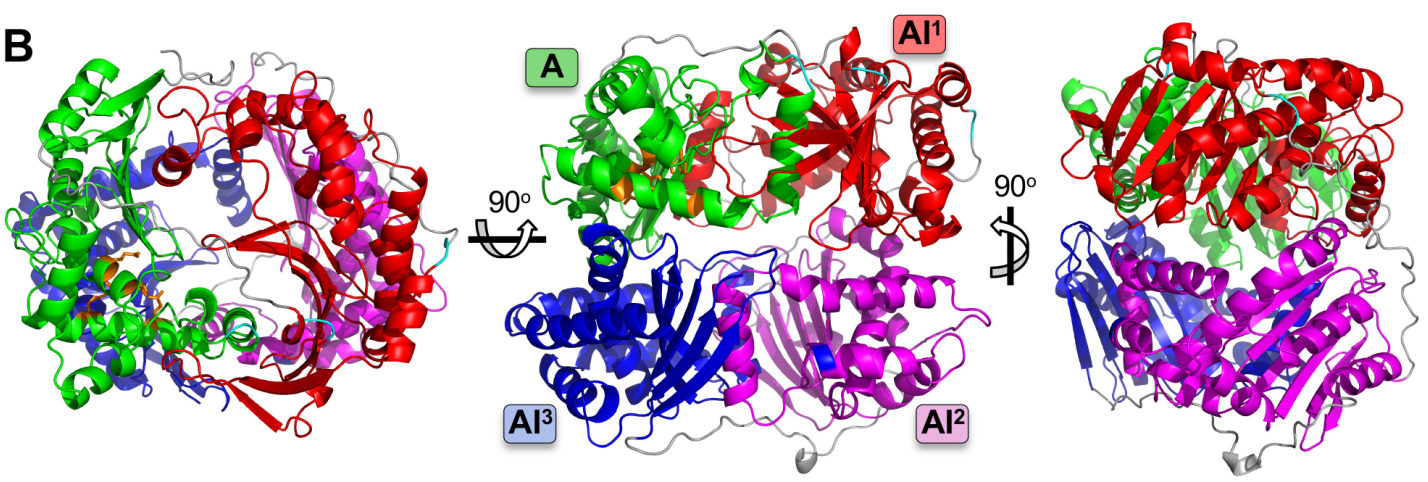

C
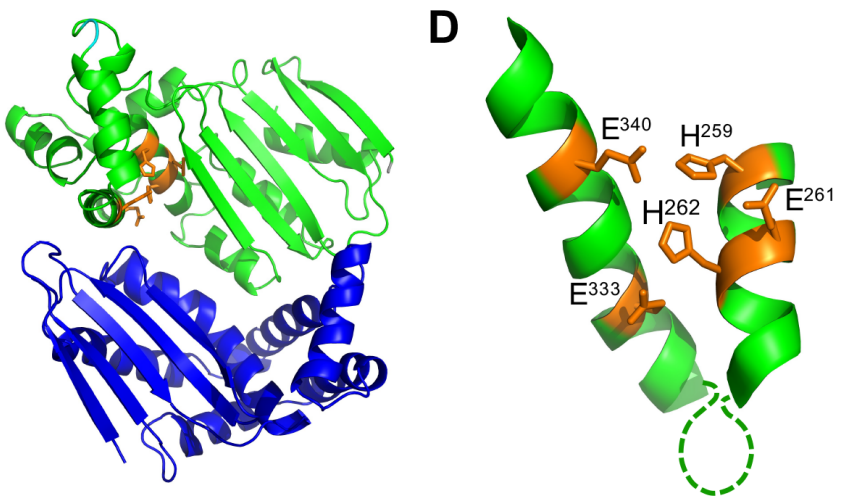

E

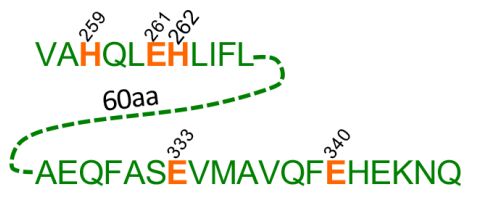

Figure 1. Structural features of TLN4. A) Domain structure of TLN4 illustrating its possession

of a signal peptide (SP), and based on the IDE structure, a single active domain (A), 3 inactive

domains $\left(\mathrm{IA}^{1-3}\right)$, and a $\mathrm{C}$-terminal repeat domain. Note that a largely featureless region between

$\mathrm{IA}^{3}$ and the repeat domain is not shown and is instead denoted by //. Numbering indicates amino acid positions and is based on the sequenced cDNA of TLN4 (22). B) Structural model of TLN4 showing the arrangement of domains into a shell surrounding a central catalytic chamber. Three different views are provided. C) The $\mathrm{A}$ and $\mathrm{AI}^{3}$ domains viewed from the center of the chamber with resides that coordinate zinc binding shown in orange. D) Zoom in of the adjacent alpha helices of the A domain showing the residues (orange) that are predicted to coordinate binding to zinc. E) Sequence of the alpha helices shown in D) including residues (orange) that likely mediate binding to zinc as a cofactor for proteolytic activity. 
A

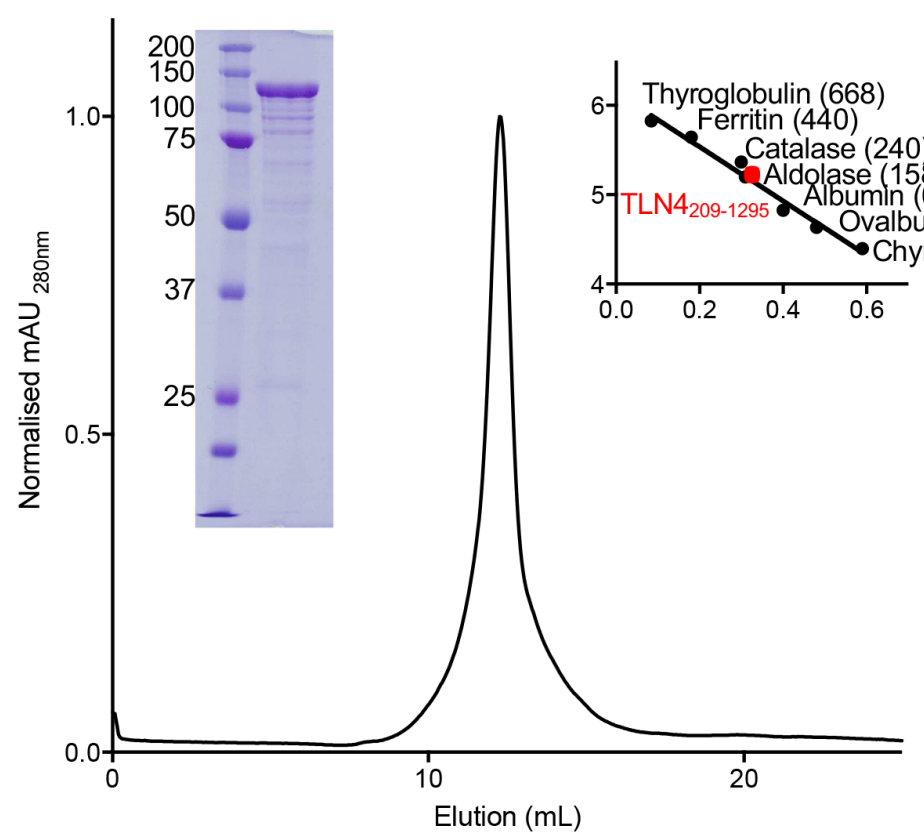

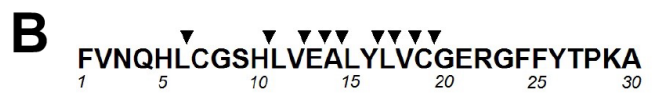

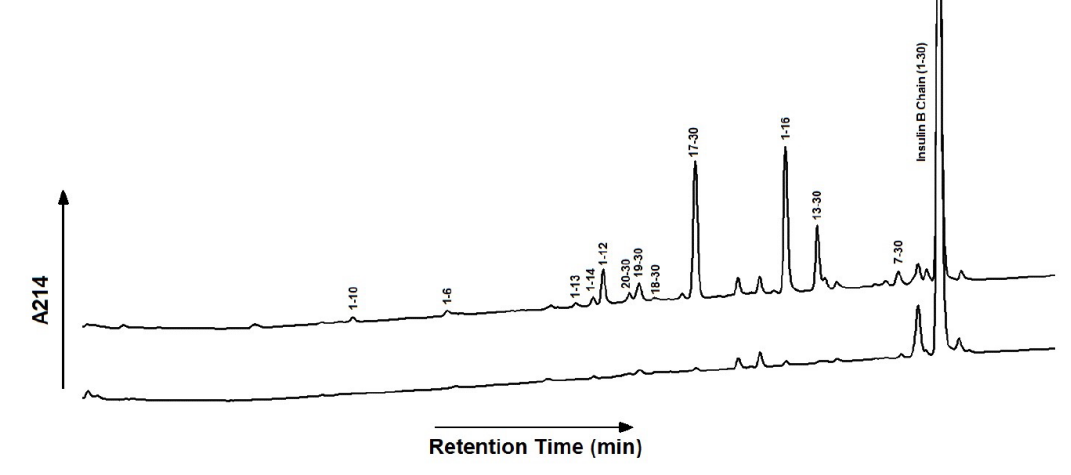

\begin{tabular}{|c|c|c|c|}
\hline $\begin{array}{c}\text { Insulin B Chain } \\
\text { fragment }\end{array}$ & Expected Mass & Observed Mass & Cleavage Site \\
\hline $1-10$ & 1140.48 & 1140.51 & $\mathrm{~F}^{1}-\mathrm{H}^{10}$ \\
\hline $1-6$ & 756.40 & 756.39 & $F^{1}-L^{6}$ \\
\hline $1-13$ & 1481.68 & 1481.71 & $F^{1}-E^{13}$ \\
\hline $1-14$ & 1552.71 & 1552.75 & $\mathrm{~F}^{1}-\mathrm{A}^{14}$ \\
\hline $1-12$ & 1352.65 & 1352.67 & $F^{1}-V^{12}$ \\
\hline $20-30$ & 1271.63 & 1271.63 & $\mathrm{G}^{20}-\mathrm{A}^{30}$ \\
\hline $19-30$ & 1374.63 & 1374.64 & $C^{19}-A^{30}$ \\
\hline $18-30$ & 1473.69 & 1473.71 & $V^{18}-A^{30}$ \\
\hline $17-30$ & 1586.79 & 1589.79 & $L^{17}-A^{30}$ \\
\hline $1-16$ & 1828.67 & 1828.89 & $F^{1}-Y^{16}$ \\
\hline $13-30$ & 2062.97 & 2063.02 & $E^{13}-A^{30}$ \\
\hline $7-30$ & 2659.24 & 2756.24 & $C^{7}-A^{30}$ \\
\hline $1-30$ & 3493.67 & 3493.51 & (insulin B chain) \\
\hline
\end{tabular}

369 Figure 2. Recombinant TLN4 proteolytic activity using $\beta$-insulin as a model substrate. A)

370 Chromatogram showing the elution of recombinant TLN4 as a monodispersed peak from size 
371 exclusion chromatography. SDS-PAGE of purified TLN4 is shown inset on the left, with

372 molecular weight markers indicated on the right of elution peak. Values in brackets indicate

373 molecular weight in kDa. B) HPLC profile of insulin B chain cleaved by TLN4. Hydrolysis

374 products were determined by comparing a reaction allowed to incubate (top line) versus a

375 reaction that was immediately stopped (bottom line). Captured peaks are labeled with their

376 residue number and cleavage map was developed (inset). C) Mass spectrometry analysis of

377 captured hydrolysis products from Insulin B Chain are shown with their expected mass and

378 observed mass along with the fragment and cleavage sites that each product represents. 

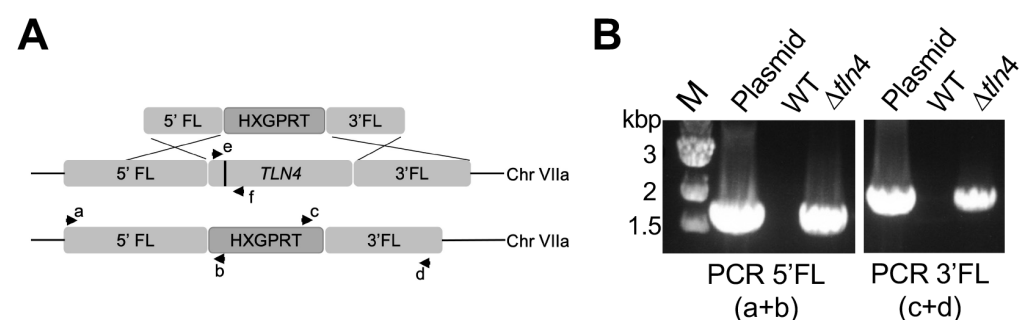

$(a+b)$

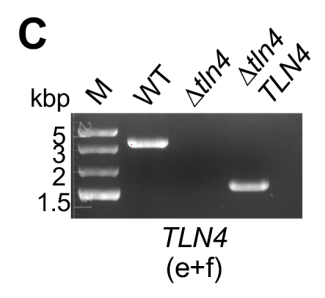

$(\mathrm{c}+\mathrm{d})$
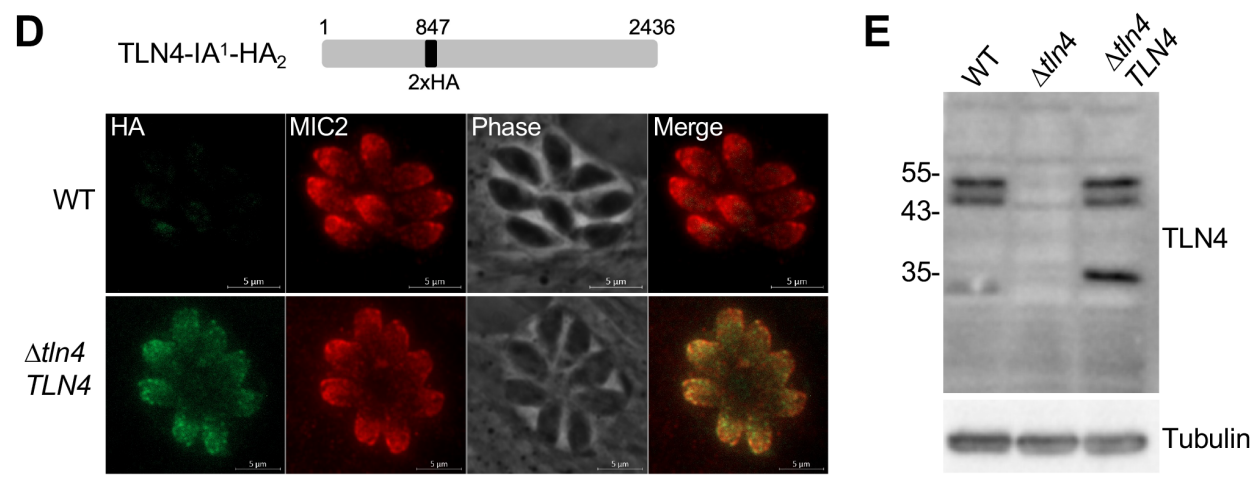

Figure 3. Genetic disruption of TLN4. A) Schematic showing the TLN4 genomic locus and homologous recombination with a cassette containing a TLN4 5' flank, the HXGPRT selectable marker, and a TLN4 3'flank. Arrows and letters indicate the placement of primers used in panel $\mathrm{B}$, the black bar in TLN4 indicates an intron that permits distinction of the gene from the cDNA in panel C. Other introns of TLN4 are not shown. B) PCR validation of selectable marker integration at the 5' (primers a+b) and 3' (primers $\mathrm{c}+\mathrm{d}$ ) end. M, molecular weight marker. C) PCR validation of $T L N 4$ deletion $(\Delta t \ln 4)$ and complementation $(\triangle t \ln 4 T L N 4)$ with the cDNA

386 (primers e+f). The differences in size are due to the presence of an intron in the WT genomic DNA versus the cDNA in the complement strain lacking the intron. D) Schematic showing the complementation construct with a 2xHA tag in the full-length TLN4 coding sequence. Immunofluorescence panel shows the micronemal localization of the complemented strain, costained with anti-MIC2. E) Mouse antibodies to TLN4 detect the $\sim 55 \mathrm{kDa}$ doublet and the $\sim 32$

$391 \mathrm{kDa}$ of TLN4 in the lysates of WT and the $\Delta t \ln 4 T L N 4$. Slower mobility of the smallest band in 392 the complement strain is due to the HA tag. Sizes (in kiloDaltons) of molecular weight markers 393 are shown to the left. Detection of T. gondii tubulin was included as a loading control. 
A
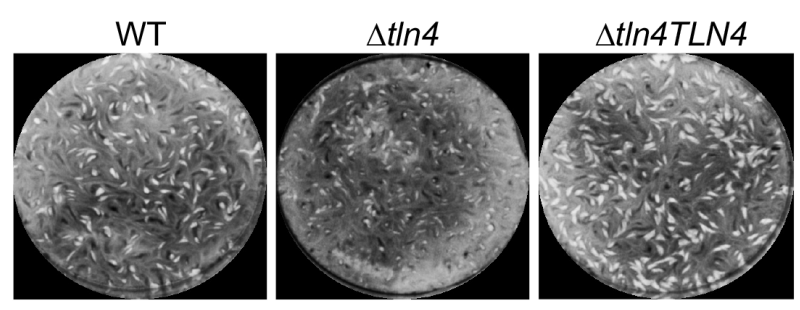

B

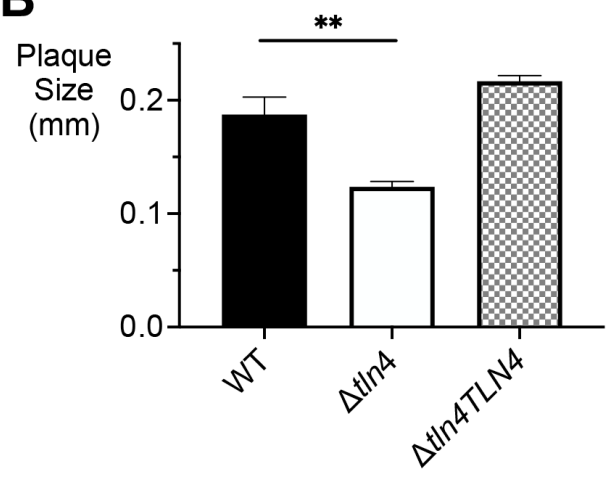

E

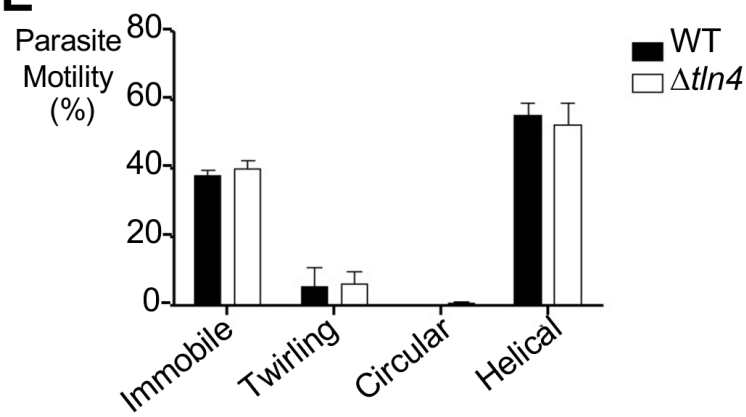

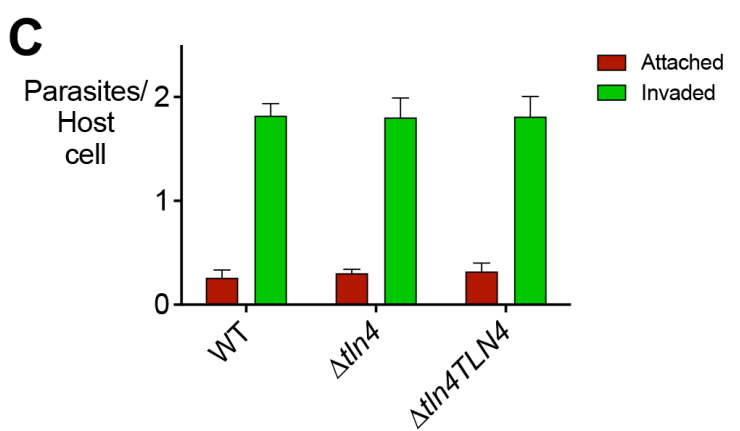

D

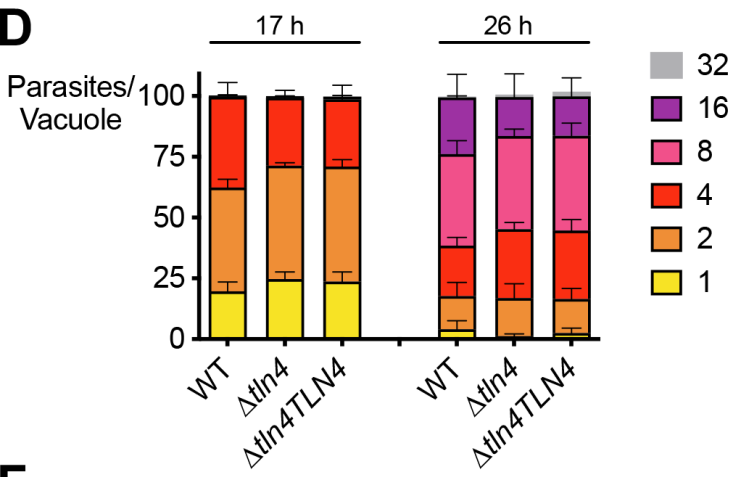

$\mathbf{F}$

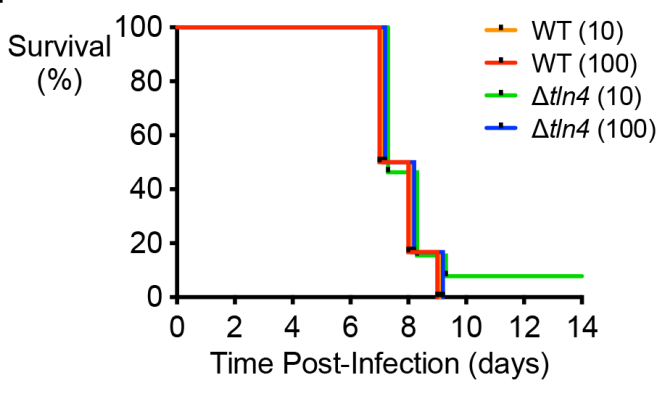

Figure 4. Effect of TLN4 deficient parasites on the lytic cycle. A) Plaque assays show smaller

395 plaques in $\Delta t \ln 4$ parasites. One hundred parasites of each strain were inoculated into 6-well

396 plates and allowed to grow for 7 days undisturbed. Wells were stained with crystal violet. B)

397 Individual plaque sizes were measured using ImageJ. Data represent three independent experiments with triplicates within each experiment. C) Parasites lacking TLN4 show normal

399 invasion. HFF cells in 8-well chamber slides were inoculated with parental, $\Delta t \ln 4$, or $\Delta t \ln 4 T L N 4$

400 parasites and allowed to invade for $20 \mathrm{~min}$ prior to fixation. Wells were differentially stained

401 with SAG1 antibodies to detect attached or invaded parasites per host cell nucleus. D) Parasites

402 lacking TLN4 replicate normally. Parasites were inoculated into 8-well chamber slides and

403 allowed to replicate for $17 \mathrm{~h}$ or $26 \mathrm{~h}$ prior to fixation and enumeration of parasites per vacuole. 
404 Data in invasion and replication graphs represent means \pm SEM of three independent

405 experiments each with triplicate samples. E) $\Delta \mathrm{t} \ln 4$ parasites show normal modes of gliding

406 motility. Video microscopy to enumerate the types of gliding motility show the percentages of

407 immobile parasites, and parasites performing twirling, circular, and helical gliding in all strains

408 tested. Graphs indicate the means \pm SEM of three independent experiments. $* p \leq 0.05$ by

409 Student's $t$-test. F) TLN4 does not play a role in acute virulence. Swiss-Webster mice were

410 infected intraperitoneally with 10 or 100 tachyzoites of WT or $\Delta t \ln 4$, and survival time was

411 enumerated; 12 mice were infected for each strain and inocula. 

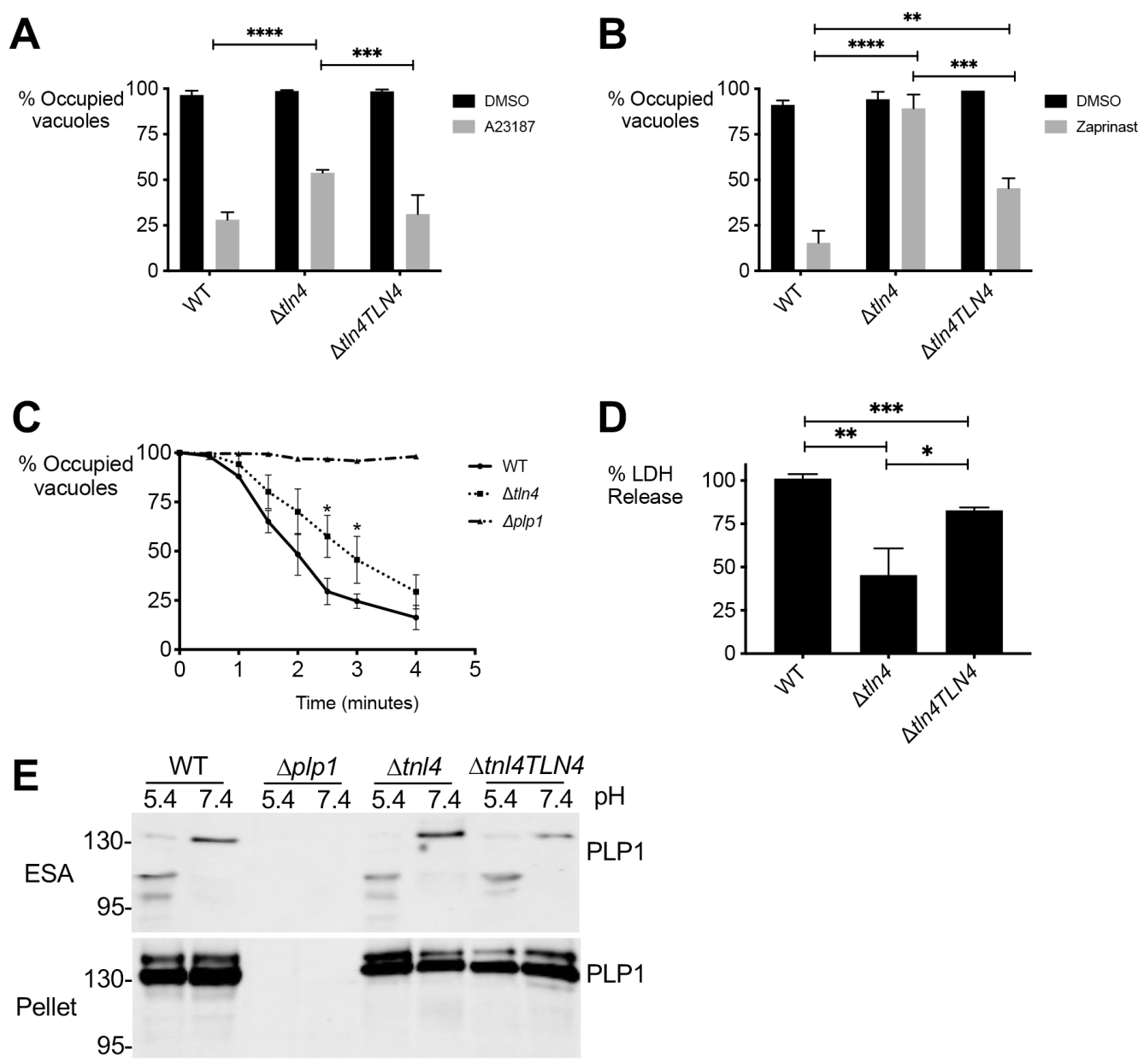

414 Figure 5. TLN4 deficient parasites are defective in egress. A) and B) Parasite egress was

415 quantified by immunofluorescence microscopy. Thirty-hour vacuoles were treated with DMSO

416 or $2 \mu \mathrm{M}$ A23187 (A) or $200 \mu \mathrm{M}$ zaprinast (B) for 5 min prior to fixation and enumeration.

$417 * p \leq 0.05, * * \mathrm{p} \leq 0.01, * * * \mathrm{p} \leq 0.001, * * * * p \leq 0.0001$ by Student's $t$-test. C) Time-course of egress

418 with $2 \mu \mathrm{M}$ A23187, quantified by immunofluorescence as in A and B. $\Delta p l p 1$ parasites were

419 included as a negative control for egress. D) Lactate dehydrogenase (LDH) release following

420 induction with $100 \mu \mathrm{M}$ zaprinast was used as a measure of egress. Data are normalized to WT

421 LDH release. All graphs shown are means \pm SEM from three biological replicates each with

422 triplicate samples. E) Parasites lacking TLN4 has normal PLP1 processing. Pellet and ESA were

423 collected from parasites at pH 5.4 and 7.4, separated on SDS-PAGE, blotted onto membranes, 
424 and probed with rabbit anti-PLP1 antibodies. Processing of PLP1 in the ESA of all strains

425 expressing PLP1 observed at pH 5.4. The pellet blot (bottom) acts as a loading control.

427 References

428 1. Rock KL, Kono H. 2008. The inflammatory response to cell death. Annu Rev Pathol 3:99$429 \quad 126$.

2. Xia J, Cheng X-Y, Wang X-J, Peng H-J. 2017. Association between Toxoplasma gondii types and outcomes of human infection: A meta-analysis. Acta Microbiol Immunol Hung Host Cells by Toxoplasma gondii. Annu Rev Microbiol 73:579-599. egress and host-cell invasion. EMBO J 31:4524-4534.

5. Wiersma HI, Galuska SE, Tomley FM, Sibley LD, Liberator PA, Donald RG. 2004. A role for coccidian cGMP-dependent protein kinase in motility and invasion. Int J Parasitol Arrizabalaga G. 2012. A forward genetic screen reveals that calcium-dependent protein

$442 \quad$ kinase 3 regulates egress in Toxoplasma. PLoS Pathog 8:e1003049. 
445 8. McCoy JM, Whitehead L, Dooren GG van, Tonkin CJ. 2012. TgCDPK3 regulates calcium-

446 dependent egress of Toxoplasma gondii from host cells. PLoS Pathog 8:e1003066.

447 9. Carruthers VB, Giddings OK, Sibley LD. 1999. Secretion of micronemal proteins is

$448 \quad$ associated with Toxoplasma invasion of host cells. Cell Microbiol 1:225-235.

449 10. Carruthers VB, Moreno SN, Sibley LD. 1999. Ethanol and acetaldehyde elevate

450 intracellular $[\mathrm{Ca} 2+]$ and stimulate microneme discharge in Toxoplasma gondii. Biochem $\mathrm{J}$

$451 \quad 342($ Pt 2):379-386.

452 11. Carruthers VB, Sibley LD. 1999. Mobilization of intracellular calcium stimulates

453 microneme discharge in Toxoplasma gondii. Mol Microbiol 31:421-428.

454 12. Lovett JL, Marchesini N, Moreno SN, Sibley LD. 2002. Toxoplasma gondii microneme

455 secretion involves intracellular $\mathrm{Ca}(2+)$ release from inositol 1,4,5-triphosphate

$456 \quad(\mathrm{IP}(3))$ /ryanodine-sensitive stores. J Biol Chem 277:25870-6.

457 13. Kafsack BF, Pena JD, Coppens I, Ravindran S, Boothroyd JC, Carruthers VB. 2009. Rapid membrane disruption by a perforin-like protein facilitates parasite exit from host cells. Science 323:530-533.

14. Roiko MS, Svezhova N, Carruthers VB. 2014. Acidification Activates Toxoplasma gondii $461 \quad$ Motility and Egress by Enhancing Protein Secretion and Cytolytic Activity. PLoS Pathog 10:e1004488.

15. Roiko MS, Carruthers VB. 2013. Functional Dissection of Toxoplasma gondii Perforin-like

464 Protein 1 Reveals a Dual Domain Mode of Membrane Binding for Cytolysis and Parasite $465 \quad$ Egress. J Biol Chem 288:8712-8725. 
16. Bisio H, Lunghi M, Brochet M, Soldati-Favre D. 2019. Phosphatidic acid governs natural egress in Toxoplasma gondii via a guanylate cyclase receptor platform. Nat Microbiol $4: 420-428$.

17. LaFavers KA, Marquez-Nogueras KM, Coppens I, Moreno SNJ, Arrizabalaga G. 2017. A novel dense granule protein, GRA41, regulates timing of egress and calcium sensitivity in Toxoplasma gondii. Cell Microbiol 19:10.1111/cmi.12749. Epub 2017 May 17.

18. Okada T, Marmansari D, Li Z, Adilbish A, Canko S, Ueno A, Shono H, Furuoka H, Igarashi M. 2013. A novel dense granule protein, GRA22, is involved in regulating parasite

19. Pszenny V, Ehrenman K, Romano JD, Kennard A, Schultz A, Roos DS, Grigg ME, Carruthers VB, Coppens I. 2016. A Lipolytic Lecithin:Cholesterol Acyltransferase Secreted egress in Toxoplasma gondii. Mol Biochem Parasitol 189:5-13.

20. Schultz AJ, Carruthers VB. 2018. Toxoplasma gondii LCAT Primarily Contributes to by Toxoplasma Facilitates Parasite Replication and Egress. J Biol Chem 291:3725-3746. opportunistic pathogen Toxoplasma gondii deploys a diverse legion of invasion and survival proteins. J Biol Chem 280:34233-34244. 177:49-56. 
23. Sidik SM, Huet D, Ganesan SM, Huynh MH, Wang T, Nasamu AS, Thiru P, Saeij JP, Carruthers VB, Niles JC, Lourido S. 2016. A Genome-wide CRISPR Screen in Toxoplasma Identifies Essential Apicomplexan Genes. Cell 166:1423-1435.e12.

24. Hajagos BE, Turetzky JM, Peng ED, Cheng SJ, Ryan CM, Souda P, Whitelegge JP, Lebrun M, Dubremetz JF, Bradley PJ. 2012. Molecular dissection of novel trafficking and processing of the Toxoplasma gondii rhoptry metalloprotease toxolysin-1. Traffic Cph Den

25. Shen Y, Joachimiak A, Rosner MR, Tang W-J. 2006. Structures of human insulin13:292-304.

26. McRobert L, Taylor CJ, Deng W, Fivelman QL, Cummings RM, Polley SD, Billker O, Baker DA. 2008. Gametogenesis in Malaria Parasites Is Mediated by the cGMP-Dependent Protein Kinase. PLoS Biol 6:e139.

27. Malito E, Hulse RE, Tang W-J. 2008. Amyloid $\beta$-degrading cryptidases: insulin degrading enzyme, presequence peptidase, and neprilysin. Cell Mol Life Sci 65:2574-2585. Determinants of Substrate Specificity in Human Insulin-Degrading Enzyme. Biochemistry 2018. Metallopeptidases of Toxoplasma gondii: in silico identification and gene expression. Parasite Paris Fr 25:26. 
507 30. Xu R, Feng Y, Xiao L, Sibley LD. 2021. Insulinase-like Protease 1 Contributes to

508 Macrogamont Formation in Cryptosporidium parvum. mBio 12:e03405-20,

$509 \quad /$ mbio/12/2/mBio.03405-20.atom.

510 31. Xu R, Guo Y, Li N, Zhang Q, Wu H, Ryan U, Feng Y, Xiao L. 2019. Characterization of

511 INS-15, A Metalloprotease Potentially Involved in the Invasion of Cryptosporidium

512 parvum. Microorganisms 7.

513 32. Huynh MH, Rabenau KE, Harper JM, Beatty WL, Sibley LD, V C. 2003. Rapid invasion of

514 host cells by Toxoplasma requires secretion of the MIC2-M2AP adhesive protein complex.

$515 \quad$ Embo J 22:2082-2090. 